\title{
Vacancy-Fluorine Clusters in Silicon
}

\author{
A.I. Chroneos ${ }^{a, *}$, R.V. Vovk ${ }^{b}$, I.L. Goulatis ${ }^{b}$, Z.F. Nazyrov ${ }^{b}$, V.M. Pinto Simoes ${ }^{c}$, \\ M. JANUSZCZYK ${ }^{d}$ AND J.N. LATOSIŃSKA ${ }^{d}$ \\ ${ }^{a}$ Department of Materials, Imperial College, London SW7 2AZ, United Kingdom \\ ${ }^{b}$ Kharkov National University, 4 Svoboda Sq., 61077 Kharkov, Ukraine \\ ${ }^{c}$ IPA_Instituto Superior Autónomo de Estudos Politécnicos, Rua de Xabregas, 20, 1 1900-440 Lisboa, Portugal \\ ${ }^{d}$ Adam Mickiewicz University, Umultowska 85, 61-614 Poznań, Poland
}

(Received October 21, 2010; in final form December 7, 2010)

\begin{abstract}
Fluorine $(\mathrm{F})$ doping and the formation of F-vacancy $\left(\mathrm{F}_{n} \mathrm{~V}_{m}\right)$ clusters have been extensively studied in silicon $(\mathrm{Si})$ as they can suppress the transient self-interstitial mediated diffusion of boron (B). Recent experimental studies by Bernardi et al. revealed that there is no significant concentration of $\mathrm{F}_{n} \mathrm{~V}_{m}$ clusters (for $n \geq 4, m \geq 1$ ) in disagreement with a number of density functional theory studies. In the present study we use electronic structure calculations to evaluate the binding energies of $\mathrm{F}_{n} \mathrm{~V}_{m}$ clusters and $\mathrm{V}_{n}$ clusters. The significant binding energies of the $\mathrm{V}_{n}$ clusters reveals that the concentration of the large $\mathrm{F}_{n} \mathrm{~V}_{m}$ clusters $(n \geq 4, m \geq 1)$ will be limited compared to the $\mathrm{V}_{n}$ clusters or even smaller clusters.
\end{abstract}

PACS: 31.15.es, 61.72.jd, 61.72.uf, 61.72.Yx

\section{Introduction}

Codoping in semiconductor materials is an efficient way to control the diffusion of dopants and control their electrical activation [1-6]. One of the most important materials where codoping strategies have been applied is $\mathrm{Si}[1-12] . \mathrm{F}$ atoms in Si saturate the dangling bonds of vacancies resulting in the formation of $\mathrm{F}_{n} \mathrm{~V}_{m}$ clusters, which in turn suppress the transient enhanced diffusion of B (Refs. [1-12] and references therein). It has been determined in previous experimental studies that the average number of $\mathrm{F}$ atoms trapped per vacancy is $2-3$ (see Refs. [3, 4]). Density functional theory (DFT) predictions indicate that the formation of the larger $\mathrm{F}_{n} \mathrm{~V}_{m}$ clusters is favored because of the energy gain due to the vacancy dangling-bond saturation by the $\mathrm{F}$ atoms [811]. According to a recent model and interpretation [11] of the stability of the $\mathrm{F}_{n} \mathrm{~V}_{m}$ clusters there is no limit on their size and therefore they are expected to grow until they saturate the available dangling bonds for a given temperature. Conversely, Bernardi et al. [5] could not determine a detectable concentration of $\mathrm{F}_{n} \mathrm{~V}_{m}$ clusters (for $n \geq 4, m \geq 1$ ). Nevertheless, the results of Bernardi et al. [5] are consistent with previous studies supporting the reduction of $\mathrm{B}$ transient enhanced diffusion by the codoping with F (for example Ref. [9] and references therein).

\footnotetext{
* corresponding author; e-mail:

alexander.chroneos@imperial.ac.uk
}

The impact of $\mathrm{V}_{n}$ cluster formation has not been considered in previous studies attempting to model the clustering of $\mathrm{F}$ atoms with $\mathrm{V}$. The formation of $\mathrm{V}_{n}$ clusters results in the reduction of the dangling bonds (for example a $\mathrm{V}_{2}$ pair reduces the dangling bonds to 6 compared to 8 in the case of two isolated V) and is energetically favorable [13]. The $\mathrm{V}_{n}$ are competing to $\mathrm{F}_{n} \mathrm{~V}_{m}$ clusters as they are limiting the unbound $\mathrm{V}$ concentration available for the $\mathrm{F}$ atoms to bind. In the present study we apply DFT to predict the most stable $\mathrm{F}_{n} \mathrm{~V}_{m}$ and $\mathrm{V}_{n}$ clusters in $\mathrm{Si}$, whereas by considering mass action analysis we can justify the discrepancies in the literature.

\section{Methodology \\ 2.1. Calculation details}

The simulations were performed using the DFT code CASTEP $[14,15]$ with the Perdew-Burke-Ernzerhof (PBE) generalized gradient approximation (GGA) functional [16] and ultrasoft Vanderbilt pseudopotentials [17]. A 64 site tetragonal diamond structure Si supercell, periodic boundary conditions and $2 \times 2 \times 2$ Monkhorst-Pack (MP) [18] $\boldsymbol{k}$-point sampling was used. A plane wave basis with an energy cut-off of $350 \mathrm{eV}$ was implemented. The atomic coordinates and unit-cell parameters were relaxed using energy minimization. The efficacy of this approach to describe the defect chemistry of semiconductors has been previously demonstrated [19-21].

\subsection{Approximations}

DFT calculations based upon either the GGA or the local density approximation (LDA) underestimate the formation energies of defects in $\mathrm{Si}$ and related materials. 
This is mainly due to the lack of exact exchange in these functionals [22]. The band gap of $\mathrm{Si}$ is severely underestimated due to the inappropriate description of exchange when LDA and the PW91 are used [22]. Thus the present study focuses on uncharged supercell calculations performed at GGA and PBE level of the theory. This allowed obtaining differences in energies, less affected by the systematic errors in the exchange-correlation energy. The condition of charge neutrality of supercells is full field by assuming mid-gap Fermi level at which the $\mathrm{F}_{n} \mathrm{~V}_{m}$ clusters should be neutral in charge.

The 64 atomic site supercell sufficiently describes the system as discussed in recent work of related systems in Si and other group IV semiconductors [23-31]. This is because in the 64 supercell the fluorine atoms are sufficiently separated from their periodic images. With the defect-defect interactions being very small at these distances the dopant and its periodic image interactions will not affect the results. As demonstrated by Probert and Payne [23] larger MP $k$-point grids and supercell sizes lead to small differences in the defect energies, consistently with previous work in $\mathrm{Si}$.

\section{Results and discussion}

When an interstitial $\mathrm{F}$ is positioned in-between two $\mathrm{Si}$ atoms (bond-center position), it forms two covalent $\sigma$ bonds, releasing one electron to the crystal and in a consequence becoming effectively positively charged for most conditions [9]. If we assume the tetrahedral position for the interstitial $\mathrm{F}$, then an electron is needed for the interstitial $\mathrm{F}$ to complete its outer shell. Therefore, the interstitial $\mathrm{F}$ is expected to be negatively charged. Thus we have found that bond-center position for the interstitial $\mathrm{F}$ is more energetically favourable than the tetrahedral position. This conclusion is in a good agreement with previous first-principles predictions for $\mathrm{F}$ interstitials in $\mathrm{Si}[9]$.

Cluster formation can be quantified by calculating the binding energies. The binding energy, $E_{\mathrm{b}}\left(\mathrm{F}_{n} \mathrm{~V}_{m} \mathrm{Si}_{N-n-m}\right)$ of $n \mathrm{~F}$ atoms to $m \mathrm{~V}$ to form a $\mathrm{F}_{n} \mathrm{~V}_{m}$ cluster in $\mathrm{Si}$ is given by

$$
\begin{aligned}
& E_{\mathrm{b}}\left(\mathrm{F}_{n} \mathrm{~V}_{m} \mathrm{Si}_{N-n-m}\right)=E\left(\mathrm{~F}_{n} \mathrm{~V}_{m} \mathrm{Si}_{N-n-m}\right) \\
& \quad-n E\left(\mathrm{FSi}_{N-1}\right)-m E\left(\mathrm{VSi}_{N-1}\right) \\
& \quad+(n+m-1) E\left(\mathrm{Si}_{N}\right),
\end{aligned}
$$

where $E\left(\mathrm{~F}_{n} \mathrm{~V}_{m} \mathrm{Si}_{N-n-m}\right)$ is the energy of a $N$ lattice site supercell (here $N=64$ ) containing $N-n-m \mathrm{Si}$ atoms, $n \mathrm{~F}$ atoms and $m$ vacancies, $E\left(\mathrm{FSi}_{N-1}\right)$ is the energy of a supercell containing one $\mathrm{F}$ and $N-1 \mathrm{Si}$ atoms; $E\left(\mathrm{VSi}_{N-1}\right)$ is the energy of a supercell containing one $\mathrm{V}$ and $N-1 \mathrm{Si}$ atoms; and $E\left(\mathrm{Si}_{N}\right)$ is the energy of the $N$ $\mathrm{Si}$ atom supercell. The physical meaning of a negative binding energy is that the $\mathrm{F}_{n} \mathrm{~V}_{m}$ cluster is more stable with respect to $n$ isolated $\mathrm{F}$ and $m$ isolated $\mathrm{V}$.

The energy change for every added interstitial $\mathrm{F}$ exceeds $-1.7 \mathrm{eV}$ for all the $\mathrm{F}_{n} \mathrm{~V}_{m}$ clusters considered. This is the energy difference of $E_{\mathrm{b}}\left(\mathrm{F}_{n+1} \mathrm{~V}\right)-E_{\mathrm{b}}\left(\mathrm{F}_{n} \mathrm{~V}\right)$ or $E_{\mathrm{b}}\left(\mathrm{F}_{n+1} \mathrm{~V}_{2}\right)-E_{\mathrm{b}}\left(\mathrm{F}_{n} \mathrm{~V}_{2}\right)$ see Table. Consistently with previous studies (for example Ref. [11] and references therein) of clusters with all dangling bonds saturated have the general formula $\mathrm{F}_{2 m+2} \mathrm{~V}_{m}$ (for example $\mathrm{F}_{4} \mathrm{~V}$ and $\mathrm{F}_{6} \mathrm{~V}_{2}$ ) exhibit the highest binding energies. As the bond-center F interstitials are positively charged they should repel each other. Consistently with the previous work [9] we performed calculations for the clusters in which we assumed a fully relaxed configuration (i.e. in which the $\mathrm{F}$ atoms repel each other). It is worth noting that such clusters are more energetically favourable compared to the clusters where the $\mathrm{F}$ atoms are constrained to the original dangling bond directions (for example the $\mathrm{F}_{4} \mathrm{~V}$ cluster, refer to Fig. 1).

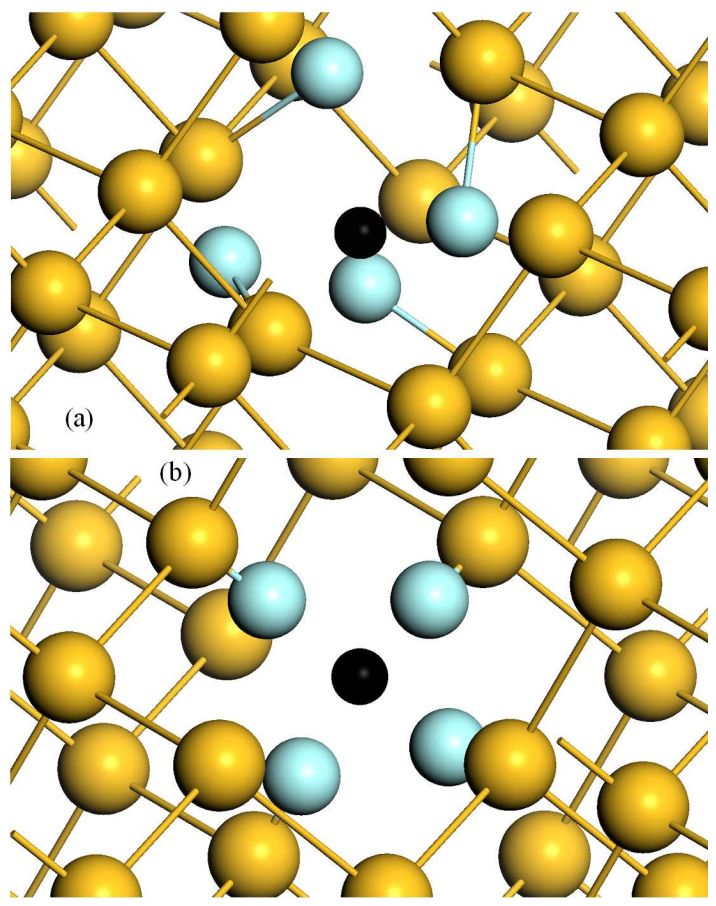

Fig. 1. The $\mathrm{F}_{4} \mathrm{~V}$ cluster in the (a) fully relaxed configuration where the $\mathrm{F}$ atoms repel each other and (b) when the $\mathrm{F}$ atoms are constrained to the original dangling bond directions. Yellow and light blue spheres represent the $\mathrm{Si}$ and $\mathrm{F}$ atoms respectively, whereas black spheres the V.

Binding energies $(\mathrm{eV})$ for $\mathrm{F}_{n} \mathrm{~V}_{m}$ clusters in $\mathrm{Si}$.

TABLE

\begin{tabular}{c|c|c|c|c|c}
\hline \hline $\begin{array}{c}\text { Defect } \\
\text { cluster }\end{array}$ & $E_{\mathrm{b}}\left(\mathrm{F}_{n} \mathrm{~V}\right)$ & $\begin{array}{c}\text { Defect } \\
\text { cluster }\end{array}$ & $E_{\mathrm{b}}\left(\mathrm{F}_{n} \mathrm{~V}_{2}\right)$ & $\begin{array}{c}\text { Defect } \\
\text { cluster }\end{array}$ & $E_{\mathrm{b}}\left(\mathrm{V}_{n}\right)$ \\
\hline $\mathrm{FV}$ & -2.23 & $\mathrm{FV}_{2}$ & -4.26 & $\mathrm{~V}_{2}$ & -1.58 \\
$\mathrm{~F}_{2} \mathrm{~V}$ & -4.20 & $\mathrm{~F}_{2} \mathrm{~V}_{2}$ & -6.92 & $\mathrm{~V}_{3}$ & -4.04 \\
$\mathrm{~F}_{3} \mathrm{~V}$ & -6.21 & $\mathrm{~F}_{3} \mathrm{~V}_{2}$ & -8.67 & $\mathrm{~V}_{4}$ & -6.41 \\
$\mathrm{~F}_{4} \mathrm{~V}$ & -8.36 & $\mathrm{~F}_{4} \mathrm{~V}_{2}$ & -10.61 & - & - \\
- & - & $\mathrm{F}_{5} \mathrm{~V}_{2}$ & -12.78 & - & - \\
- & - & $\mathrm{F}_{6} \mathrm{~V}_{2}$ & -14.94 & - & -
\end{tabular}


The relative concentrations of $\mathrm{F}_{n} \mathrm{~V}_{m}$ clusters can be quantified by applying mass action analysis [32]. Within the mass action framework the concentration of a $\mathrm{F}_{n} \mathrm{~V}_{m}$ cluster, i.e. $\left[\mathrm{F}_{n} \mathrm{~V}_{m}\right]$, relative to the concentration of unbound $\mathrm{F}$ atoms, i.e. $[\mathrm{F}]$, and the concentration of unbound $\mathrm{V}$, i.e. [V], is given by

$$
\frac{\left[\mathrm{F}_{n} \mathrm{~V}_{m}\right]}{[\mathrm{F}]^{n}[\mathrm{~V}]^{m}}=\exp \left(\frac{-E_{\mathrm{b}}\left(\mathrm{F}_{n} \mathrm{~V}_{m} \mathrm{Si}_{N-n-m}\right)}{k_{\mathrm{B}} T}\right),
$$

where $k_{\mathrm{B}}$ is Boltzmann's constant, $T$ is the temperature and $E_{\mathrm{b}}$ is the binding energy of clusters (given in Table).

Equation (2) highlights that the formation of the larger clusters is not only dependent upon the temperature and the binding energy differences between the clusters but also on the relative $[\mathrm{F}]$ and $[\mathrm{V}]$ concentrations. Using Eq. (2) one can generate a set of simultaneous equations for $\mathrm{F}_{n} \mathrm{~V}_{m}$ clusters, which can be solved using an iterative minimisation approach. The accuracy of the description through this method will rely on the accurate determination of the initial $\mathrm{V}$ concentration. The efficacy and assumptions of the presented mass action framework in related materials and issues was widely discussed in our previous papers [33-36].

Interestingly, in a recent related study concerning F-doped germanium $(\mathrm{Ge})$ mass action analysis that the really large $\mathrm{F}_{n} \mathrm{~V}_{m}$ clusters are never of significance irrespective of the relative $\mathrm{F}$ and $\mathrm{V}$ concentration conditions are irrelevant [37]. Conversely, smaller clusters such as $\mathrm{V}_{4}, \mathrm{~F}_{2} \mathrm{~V}_{2}$ and $\mathrm{FV}$ and isolated defects are more populous with their concentrations depending strongly upon the temperature [37]. Cluster populations in $\mathrm{Si}$ and $\mathrm{Ge}$ are expected to be similar given the analogous $\mathrm{F}_{n} \mathrm{~V}_{m}$ binding energies. Therefore, there is consistency with the work of Bernardi et al. [5] which could not determine a detectable concentration of $\mathrm{F}_{n} \mathrm{~V}_{m}$ clusters $(n \geq 4$, $m \geq 1)$.

\section{Conclusions}

The electronic structure calculations predict that the $\mathrm{V}_{n}$ clusters have high binding energies and can be antagonistic to $\mathrm{F}_{n} \mathrm{~V}_{m}$ clusters (Table). This is because they are limiting the unbound $\mathrm{V}$ concentration available for the $\mathrm{F}$ atoms to bind. The change in binding energy strongly depends on the number of fluorine interstitials and lattice vacancies in the defect clusters. A mass action model proposed in this paper can be applied to calculate the relative concentration of the $\mathrm{V}_{n}$ and $\mathrm{F}_{n} \mathrm{~V}_{m}$ clusters given an initially determined $\mathrm{V}$ concentration and knowledge of how much $\mathrm{F}$ is implanted in the Ge sample.

\section{Acknowledgments}

The authors acknowledge useful discussions with Robin Grimes (Imperial College London) and Hartmut Bracht (Münster University). Computing resources were provided by the HPC facility of Imperial College London.

\section{References}

[1] S.P. Jeng, T.P. Ma, R. Canteri, M. Anderle, G.W. Rubloff, Appl. Phys. Lett. 61, 1310 (1992).

[2] X.D. Pi, C.P. Burrows, P.G. Coleman, Phys. Rev. Lett. 90, 155901 (2003).

[3] P.J. Simpson, Z. Jenei, P. Asoka-Kumar, R.R. Robison, M.E. Law, Appl. Phys. Lett. 85, 1538 (2004).

[4] N.E.B. Cowern, B. Colombeau, J. Benson, A.J. Smith, W. Lerch, S. Paul, T. Graf, F. Cristiano, X. Hebras, D. Bolze, Appl. Phys. Lett. 86, 101905 (2005).

[5] F. Bernardi, J.H.R. dos Santos, M. Behar, Phys. Rev. B 76, 033201 (2007).

[6] S. Boninelli, G. Impellizzeri, S. Mirabella, F. Priolo, E. Napolitani, N. Cherkashin, F. Cristiano, Appl. Phys. Lett. 93, 061906 (2008).

[7] C.G. Van de Walle, F.R. McFeely, S.T. Pantelides, Phys. Rev. Lett. 61, 1867 (1988).

[8] M. Diebel, S.T. Dunham, Phys. Rev. Lett. 93, 245901 (2004).

[9] G.M. Lopez, V. Fiorentini, G. Impellizzeri, S. Mirabella, E. Napolitani, Phys. Rev. B 72, 045219 (2005).

[10] V. Fiorentini, G.M. Lopez, Phys. Rev. Lett. 96 , 039601 (2006).

[11] G.M. Lopez, V. Fiorentini, Appl. Phys. Lett. 89, 092113 (2006).

[12] S.A. Harrison, T.F. Edgar, G.S. Hwang, Phys. Rev. B 74, 121201 (2006).

[13] D.J. Chadi, K.J. Chang, Phys. Rev. B 38, 1523 (1988).

[14] M.C. Payne, M.P. Teter, D.C. Allan, T.A. Arias, J.D. Joannopoulos, Rev. Mod. Phys. 64, 1045 (1992).

[15] M.D. Segall, P.J.D. Lindan, M.J. Probert, C.J. Pickard, P.J. Hasnip, S.J. Clark, M.C. Payne, J. Phys., Condens. Matter 14, 2717 (2002).

[16] J. Perdew, K. Burke, M. Ernzerhof, Phys. Rev. Lett. 77, 3865 (1996).

[17] D. Vanderbilt, Phys. Rev. B 41, 7892 (1990).

[18] H.J. Monkhorst, J.D. Pack, Phys. Rev. B 13, 5188 (1976).

[19] A. Chroneos, H. Bracht, R.W. Grimes, B.P. Uberuaga, Appl. Phys. Lett. 92, 172103 (2008).

[20] H. Bracht, A. Chroneos, J. Appl. Phys. 104, 076108 (2008).

[21] A. Chroneos, H. Bracht, C. Jiang, B.P. Uberuaga, R.W. Grimes, Phys. Rev. B 78, 195201 (2008).

[22] R.J. Needs, J. Phys., Condens. Matter 11, 10437 (1999).

[23] M.I.J. Probert, M.C. Payne, Phys. Rev. B 67, 075204 (2003).

[24] A. Chroneos, C.A. Londos, J. Appl. Phys. 107, 093518 (2010).

[25] A. Chroneos, J. Appl. Phys. 107, 076102 (2010).

[26] J. Coutinho, R. Jones, P.R. Briddon, S. Öberg, Phys. Rev. B 62, 10824 (2000).

[27] A. Chroneos, Phys. Status Solidi B 244, 3206 (2007). 
[28] A. Chroneos, C. Jiang, R.W. Grimes, U. Schwingenschlögl, H. Bracht, Appl. Phys. Lett. 94, 252104 (2009).

[29] U. Schwingenschlögl, A. Chroneos, C. Schuster, R.W. Grimes, Appl. Phys. Lett. 96, 242107 (2010).

[30] A. Chroneos, C. Jiang, R.W. Grimes, U. Schwingenschlögl, H. Bracht, Appl. Phys. Lett. 95, 112101 (2009).

[31] A. Chroneos, H. Bracht, R.W. Grimes, B.P. Uberuaga, Mater. Sci. Eng. B 154, 72 (2008).

[32] F.A. Kröger, V.J. Vink, in Solid State Physics, Eds. F. Seitz, D. Turnbull, Vol. 3, Academic Press, New York 1956, p. 307.

[33] A. Chroneos, R.W. Grimes, B.P. Uberuaga, S. Brotzmann, H. Bracht, Appl. Phys. Lett. 91, 192106 (2007).
[34] A. Chroneos, R.W. Grimes, H. Bracht, B.P. Uberuaga, J. Appl. Phys. 104, 113724 (2008).

[35] A. Chroneos, R.W. Grimes, B.P. Uberuaga, H. Bracht, Phys. Rev. B 77, 235208 (2008).

[36] A. Chroneos, R.W. Grimes, H. Bracht, J. Appl. Phys. 105, 016102 (2009).

[37] A. Chroneos, R.W. Grimes, H. Bracht, J. Appl. Phys. 106, 063707 (2009). 